\title{
Wrinkling measurement of the mechanical properties of drying salt thin films
}

Giulia L. Ferretti, Manuela Nania, Omar K. Matar, João T. Cabral*

Department of Chemical Engineering, Imperial College London, London SW7 2AZ, UK

\section{ACH/Glycerol/Water glass formation study upon drying}

Aluminium Chlorohydrate $(\mathrm{ACH})$ is an inorganic salt whose structure is often described as an inorganic polymer. It comprises $\mathrm{Al}_{13}$ units, with a Keggin ion structure, which undergoes complex transformations to form larger poly-aluminium complexes. ${ }^{1,2,3,4}$ The system investigated, a 50\% dialuminium chloride pentahydroxide aqueous solution, solely forms a glass upon desiccation, which is confirmed by the powder X-ray diffraction data shown in Figure SI1, and differential scanning calorimetry (DSC) thermograms, showing characteristic glass transitions.
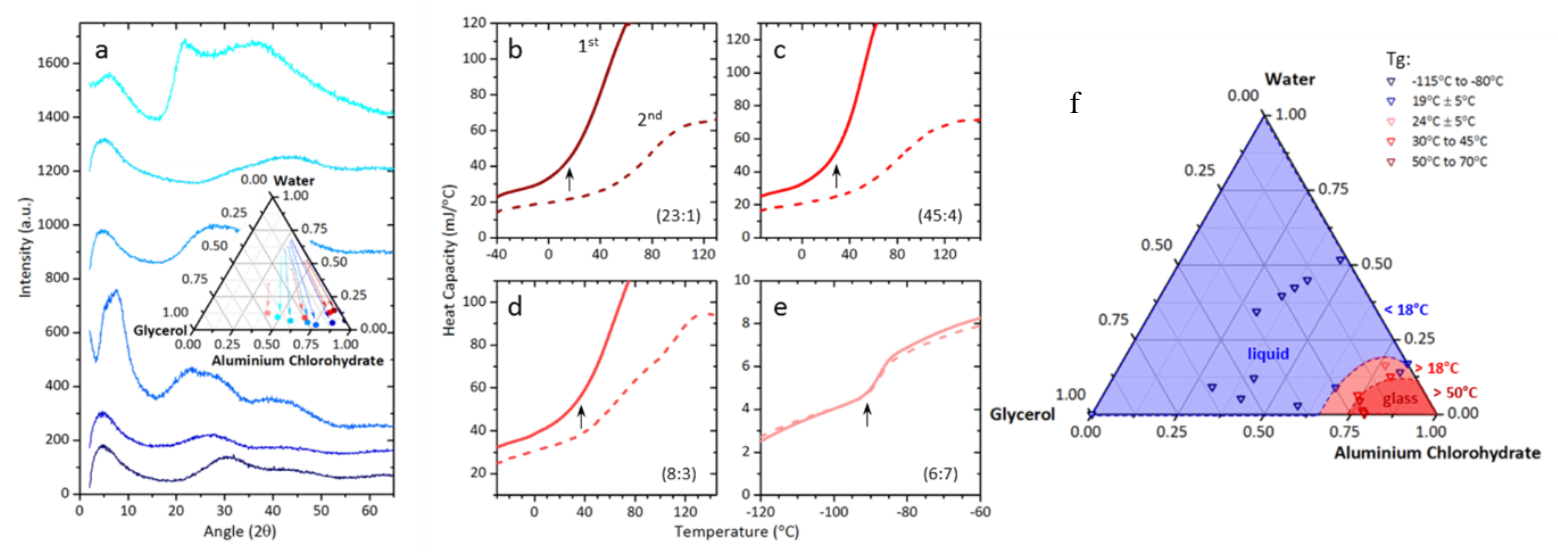

Figure SI1: (a) WAXS and (b-e) DSC of dried films at different humectant to salt ratios indicated in the ternary diagram. The solid lines correspond to the first heating ramp (up to $70^{\circ} \mathrm{C}$ for b-d), and dashed lines to second heating cycles, both carried at a rate of $10^{\circ} \mathrm{C} / \mathrm{min}$. Due to the onset of water loss around and above room temperature, $\mathrm{Tg}$ was estimated from the onset of the endotherm associated with water loss on the 1 st heating cycle for the data shown in $b$ to $d$. Onsets were determined by intersection of tangents. (f) Glass transition ranges for the ternary ACH/glycerol/water system measured by DSC. 
At sufficiently high temperatures, above the glass transition $T g$, these hygroscopic films release water, as shown in Figure SI2. Upon cycling temperature, the measured $\mathrm{Tg}$ shifts upwards, as the water content decreases. Crystallisation is not observed for this system under any conditions investigated across the composition range and drying pathway.

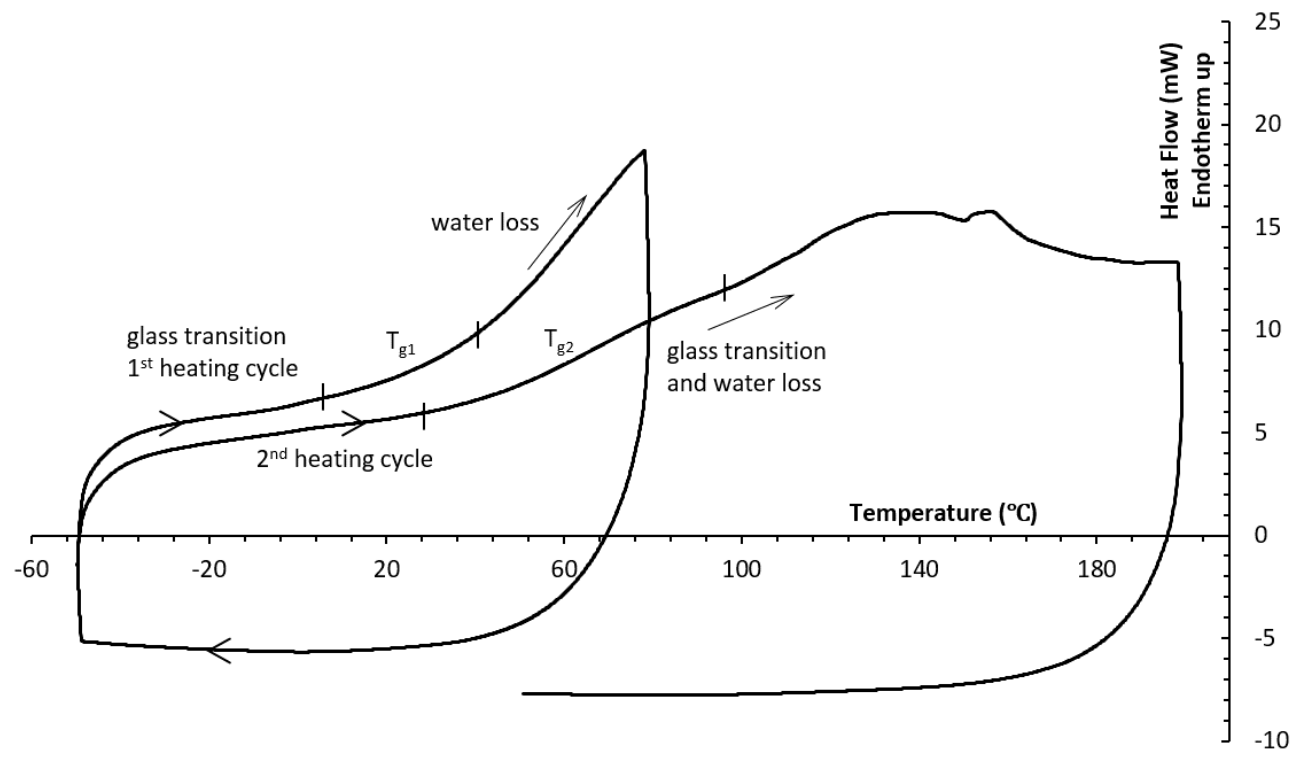

Figure SI2: Full DSC thermogram for a sample with composition of (glycerol,ACH,water) $=(0.248,0.662,0.09) \mathrm{w} / \mathrm{w}$, shown in curve (d) in Fig SI1. The large heat flow change following the glass transition represents water loss. Due to the onset water loss, the glass transition temperature shifts to higher temperatures as showed in the second cycle. No crystallisation signatures are observed.

\section{Straining method and cyclic sample straining}

The method chosen in our experiments resulted in samples exhibiting wrinkling in the relaxed state, commonly referred as ON samples. Alternatively, OFF samples wrinkle upon stretching (i.e. orthogonal compression). The procedures leading to the two distinct samples are illustrated in Figure SI3(a) OFF samples, b) ON samples). In case of OFF samples, the film is formed when the substrate is in the relaxed state and, while the procedure requires fewer steps, these samples present two main drawbacks. Firstly, cracks form in the orthogonal direction of application of tensile strain, as shown in Figure SI3(a). Secondly, as wrinkling occurs under tension, the samples need to characterised mounted on the strain stage, requiring a more complex experimental setup. We therefore opt for the procedure detailed in Figure SI3(b) or, to further eliminate crack-formation, the more laborious procedure in Figure SI3(c) requiring one sample per measurement. 


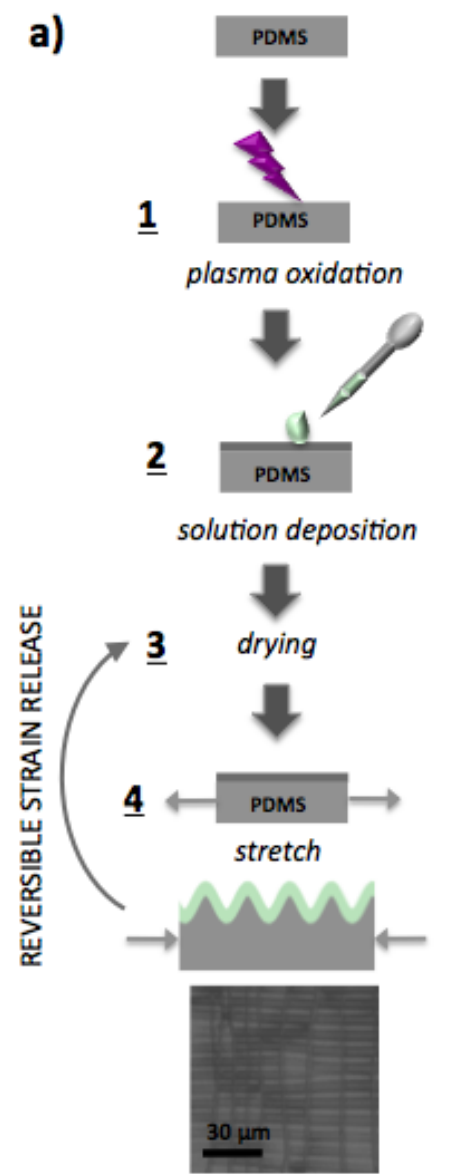

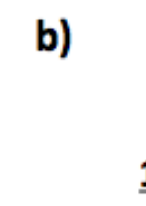
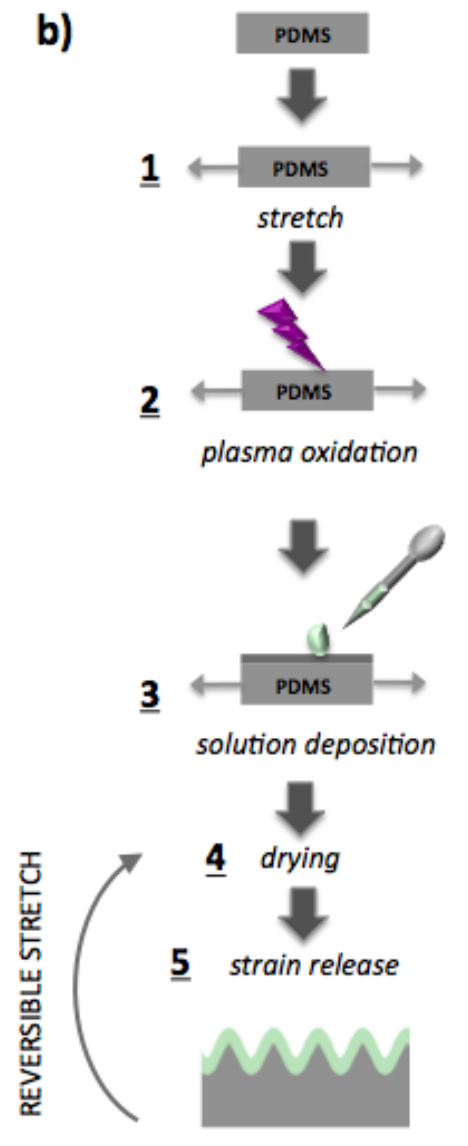

c)

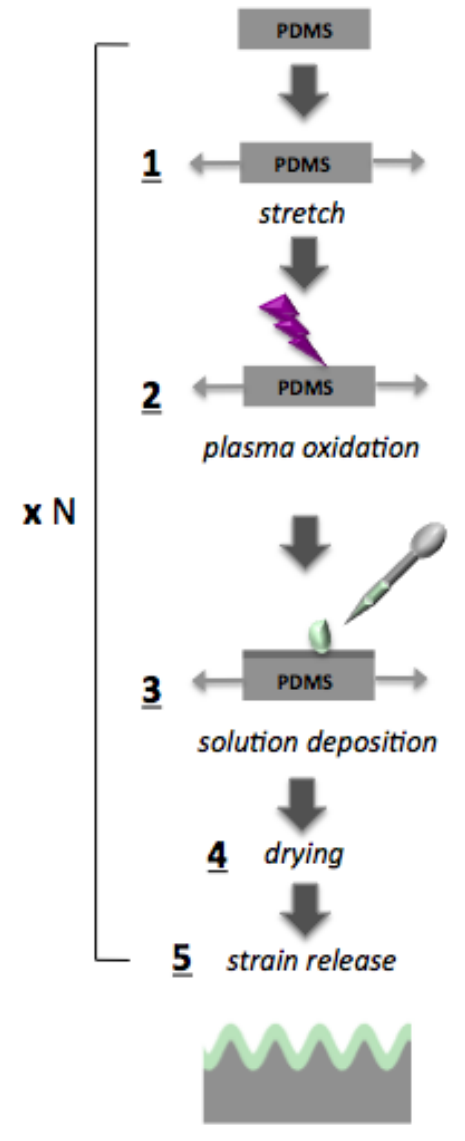

Figure SI3: Schematic of three different procedures utilised to induce wrinkling on drying film. The samples prepared through a) show wrinkles in the strained state (OFF samples). An optical microscope image of a wrinkled OFF sample is reported $\left(\mathrm{c}_{\mathrm{in}}=2 \mathrm{wt} \%, \mathrm{RH}=40 \%, \mathrm{~T}=20^{\circ} \mathrm{C}\right)$. Samples prepared following b) show wrinkles in the relaxed state, allowing $\mathrm{N}$ measurements to be made o a single sample. Procedure c) is analogous to b), except that each measurement is taken on a fresh sample, corresponding to $\mathrm{N}$ measurements on $\mathrm{N}$ samples, but further reducing surface cracking.

As shown in the schematic in Figure SI3(b), after a certain drying time, strain is released and sinusoidal wrinkles are observed in a certain range of conditions, whose characteristic dimensions can be measured and then directly related to the mechanical properties of the drying film. Afterwards, the sample can be stretched again and allowed to dry further until the desired time, at which point the strain can be released and the elastic modulus computed again through the measurement of pattern dimensions. Therefore, the mechanical characterisation of thin drying films can be carried by taking $\mathrm{N}$ measurements on a single sample via reversibly releasing and applying the same strain during drying (Figure SI3(b)). Alternatively, it is possible to by analyse $\mathrm{N}$ samples, each one allowed to dry for different time intervals (Figure SI3(c)). The results reported in the paper refer to $\mathrm{N}$ samples.

Next, we consider the wrinkling wavelength on a selected sample during drying, shown in Figure SI4. The sample was kept in the strain stage and quantitative information was extracted using optical microscopy. Removing the sample from the strain stage decreases the measurement accuracy, due to the difficulty of repositioning the PDMS coupon in the same exact initial position. Additionally, as expected, stretching the sample multiple times during drying promotes the formation of cracks. 

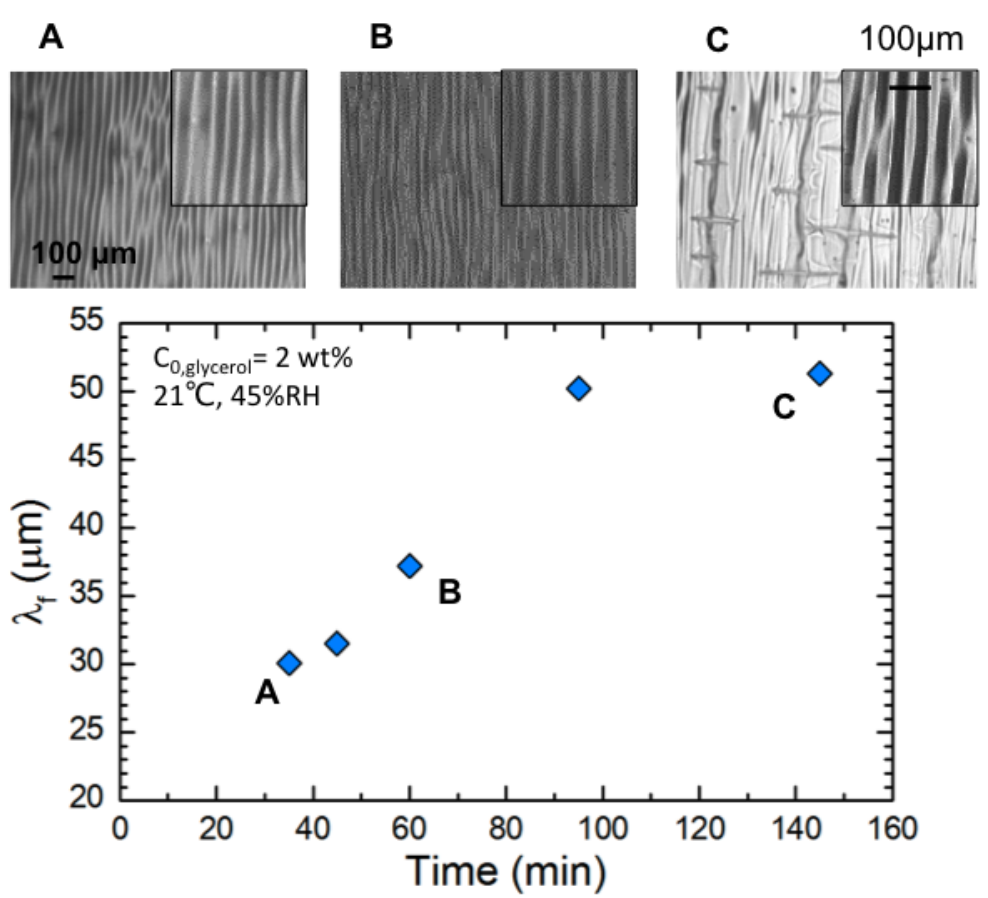

Figure SI4: Wrinkling wavelength observed on a single film as a function of drying time for a $15 \mathrm{wt} \%$ ACH and $2 \mathrm{wt} \%$ glycerol aqueous film at $21^{\circ} \mathrm{C}$ and $45 \% \mathrm{RH}$. The data refers to the same sample, stretched back to the same intial amount of $20 \%$ after each wrinkling measurement. The figure also shows optical microscope images of the wrinkled film at different stages of drying.

\section{Choice of the prestrain applied to induce wrinkling}

In our experiments, the solution was cast onto oxidised PDMS substrates, prestretched by $\varepsilon \approx 20 \%$ by means of a uniaxial mechanical strain stage. Within the sinusoidal wrinkling regimes, a series of experiments were carried in order to compute the dependence of pattern morphology on the prestrain used. A representative system with $\mathrm{c}_{0 \text {,glycerol }}=2 \mathrm{wt} \%$ was selected for this evaluation and allowed to dry for $12 \mathrm{~min}$ at $25{ }^{\circ} \mathrm{C}$ and $25 \% \mathrm{RH}$. The wrinkling amplitude, which depends strongly on prestrain, was plotted as a function of $\varepsilon$, as shown in Figure SI5. The measurement showed that a critical value of $\varepsilon_{\mathrm{c}} \approx 15 \%$ had to be exceeded in order for wrinkling to occur. Thereafter, an increase in $\varepsilon$ results in an increase in pattern amplitude. In our measurements, we typically employed a constant value of the prestrain of $\varepsilon \approx 20 \%$, which was found to exceed $\varepsilon_{\mathrm{c}}$ within the accessible experimental window. In order to reduce sample cracking, lower values of prestrain $\varepsilon$ were also evaluated, as detailed below. 


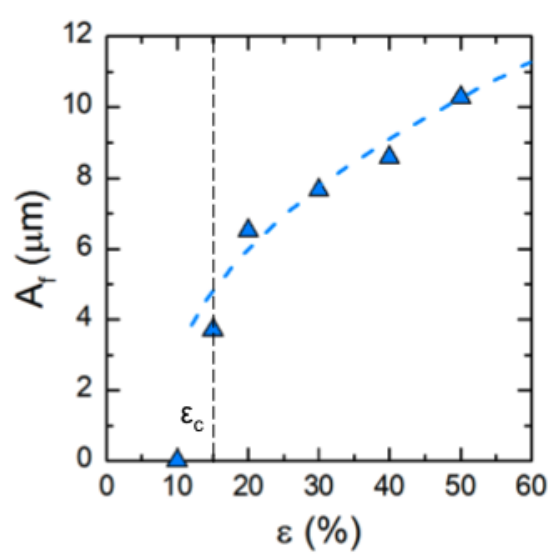

Figure SI5: Wrinkling amplitude on the film surface as a function of PDMS substrate pre-strain for a $15 \mathrm{wt} \% \mathrm{ACH}$ and $2 \mathrm{wt} \%$ glycerol aqueous film at $12 \mathrm{~min}$ drying time, $25^{\circ} \mathrm{C}$, and $25 \% \mathrm{RH}$. The line represent the fit according to eq. (1c) of the main paper.

The value of $\varepsilon_{\mathrm{c}}$ can be computed from the measured wrinkling wavelength and amplitude as a function of drying time, following eq. (1) of the main paper. Figure SI6(a) shows how the critical strain decreases with drying time, as the film hardens. After approximately $2 \mathrm{~h}$ drying time, we are no longer able to induce wrinkling, and thus infer the film's mechanical properties, as cracking and delamination dominate. In order to minimize this effect, wrinkling experiments on dry films $(3 \mathrm{~h})$ were performed at $\varepsilon=5 \%, 10 \%$, and $20 \%$, exceeding $\varepsilon_{\mathrm{c}}$, Cracking and delamination was observed at all conditions, as shown in Figure SI6(b), with an increasing spatial density, with increasing $\varepsilon$. A combination of cracks and (orthogonal) delamination is visible on the top and cross-sectional views of Figure SI6(b). Reducing $\varepsilon$ is thus not a viable approach to extending the range of application of this time-resolved wrinkling approach, likely because the modulus of the film increases abruptly near $T g$ and the adhesion between film and substrate becomes poorer at the final drying stages. Reducing film thickness would be effective at extending the experimental window, but alter drying kinetics outside region of interest.

a)

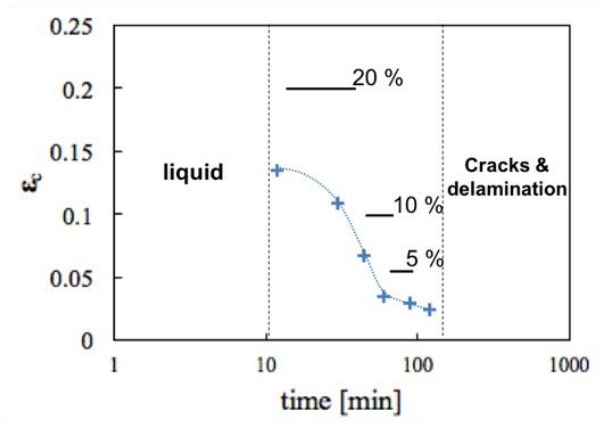

b)
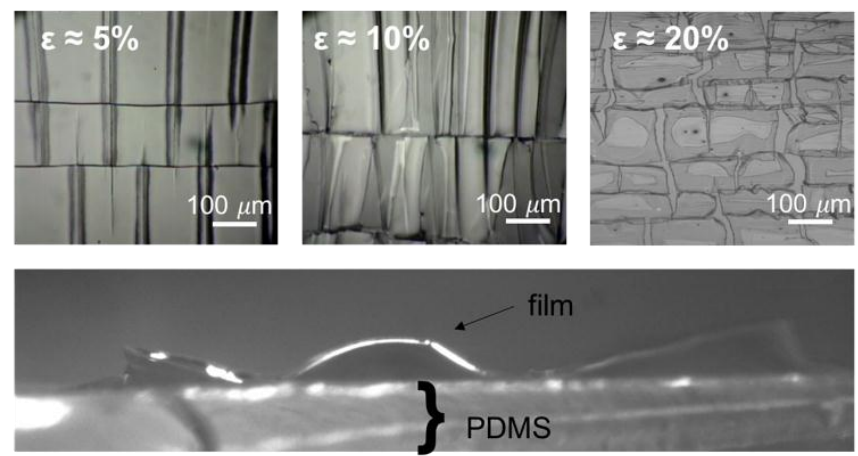

Figure SI6: a) Critical strain for wrinkling as a function of drying time, for a film with $\mathrm{c}_{0, \text { glycerol }}=2 \mathrm{wt} \%$ at $25{ }^{\circ} \mathrm{C}$ and $25 \%$ $\mathrm{RH}$. The value of strain $\varepsilon=20 \%$ chosen in the experiments is above the critical strain at all relevant drying times. b) Optical microscopy images of films with $\mathrm{c}_{0, \mathrm{glycero}}=2 \mathrm{wt} \%$ allowed to dry for 3 hours, with a prestrain of $5 \%, 10 \%$ and $20 \%$. At such long drying times, even a lower value of strain (e.g. $5 \%$ ) could not prevent delamination and cracking, hindering mechanical measurement via wrinkling. 
For this system, under the environmental conditions studied, the window of applicability of the wrinkling approach is thus approximately [12-120 min]. At lower times, the film is liquid, while at longer times, cracking and delamination dominate. Under the latter conditions, but not the former, AFM nanoindentation can still be employed, as shown in Figure SI7. This technique is, however, slower, may suffer from substrate-convolution and the accurate analysis is complex.

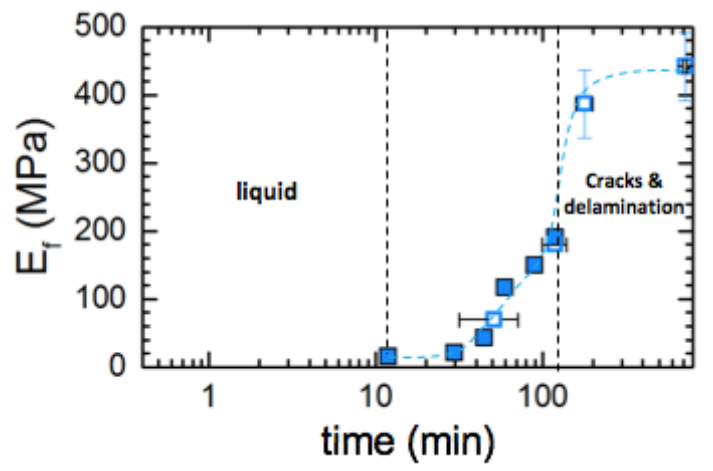

Figure SI7: Evolution of drying film mechanical properties as a function of time $\left({ }_{\mathrm{c} 0 \text {,glycerol }}=2 \mathrm{wt} \%, \mathrm{~T}=25{ }^{\circ} \mathrm{C}\right.$ and $\left.\mathrm{RH}=25 \%\right)$. The symbol ( $\square$ ) is utilised for the values obtained via wrinkling experiments using the bilayer model, while ( $\square$ ) indicates values obtained via AFM nanoindentation.

\section{Control wrinkling experiments for neat oxidised PDMS (in the absence of liquid film)}

PDMS oxidation by plasma and UVO is required for aqueous film wetting but also results in the formation of a glassy skin. As a result, this sandwich bilayer may also wrinkle upon compression, potentially affecting the measurement of interest of the mechanical properties of the drying film. We have therefore carried out careful control experiments on the wrinkling of neat oxidised PDMS to evaluate possible interference of this additional glassy layer. We have considered PDMS oxidation by both air plasma (Table SI1, Figure SI8, SI9) and UVO (Table SI2, Figure SI10), as detailed in the main paper.

\section{Wrinkling experiments on plasma oxidised PDMS}

\begin{tabular}{|c|c|c|}
\hline Time $[\mathbf{s}]$ & $\boldsymbol{\lambda}[\boldsymbol{\mu m}]$ & $\mathbf{A}[\boldsymbol{\mu m}]$ \\
\hline 90 & 0.121 & 0.009 \\
\hline 120 & 0.143 & 0.011 \\
\hline 600 & 0.719 & 0.145 \\
\hline 2400 & 2.698 & 0.809 \\
\hline 3600 & 3.398 & 1.017 \\
\hline
\end{tabular}

Table SI1: Wavelength and amplitude values on neat PDMS oxidised by means of air plasma exposure, for different time intervals ( $\mathrm{p}=7.16 \mathrm{~W}, \mathrm{P}=1 \mathrm{mbar}$ ) at $\varepsilon=20 \%$. 

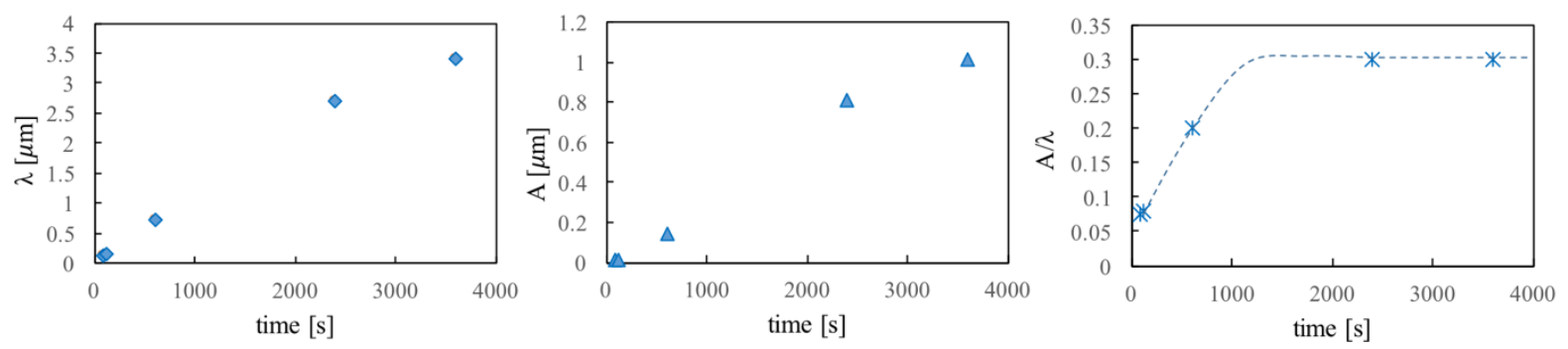

Figure SI8: Wrinkling wavelength, amplitude and aspect ratio (A/ $\lambda$ ), for PDMS oxidised by means of air plasma exposure, as a function of time $(\mathrm{p}=7.16 \mathrm{~W}, \mathrm{P}=1 \mathrm{mbar})$. The aspect ratio reaches a constant value at exposure time $\mathrm{t}_{\mathrm{ex}} \approx 1500 \mathrm{~s}$, corresponding to the saturation of the glassy layer profile.,

a)

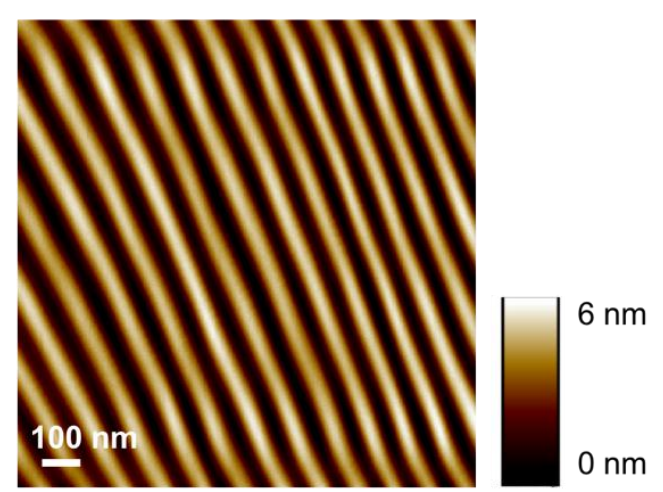

b)

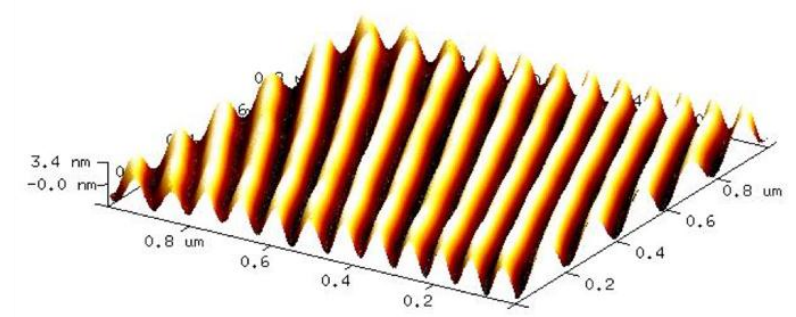

Figure SI9: AFM 2D(a) and 3D (b) images of the smallest wrinkles observed on PDMS oxidised by means of air plasma exposure, with $\mathrm{t}=90 \mathrm{~s} \mathrm{p}=7.16 \mathrm{~W}$ and $\mathrm{P}=1.3$ mbar. Periodicties $\lambda \approx 100 \mathrm{~nm}$ are readily attained at short exposure times and power, at high air pressure. ${ }^{6}$

\section{Wrinkling experiments on UVO oxidised PDMS}

\begin{tabular}{|c|c|c|}
\hline Time [min] & $\boldsymbol{\lambda}[\boldsymbol{\mu \mathbf { m } ]}$ & $\mathbf{A}[\boldsymbol{\mu m}]$ \\
\hline 60 & 30.59 & 5.5 \\
\hline 90 & 52.76 & 9.93 \\
\hline 120 & 79.8 & 15.54 \\
\hline
\end{tabular}

Table SI2: Wavelength and amplitude values of wrinkles on PDMS oxidised by means of UVO exposure, for different time intervals.
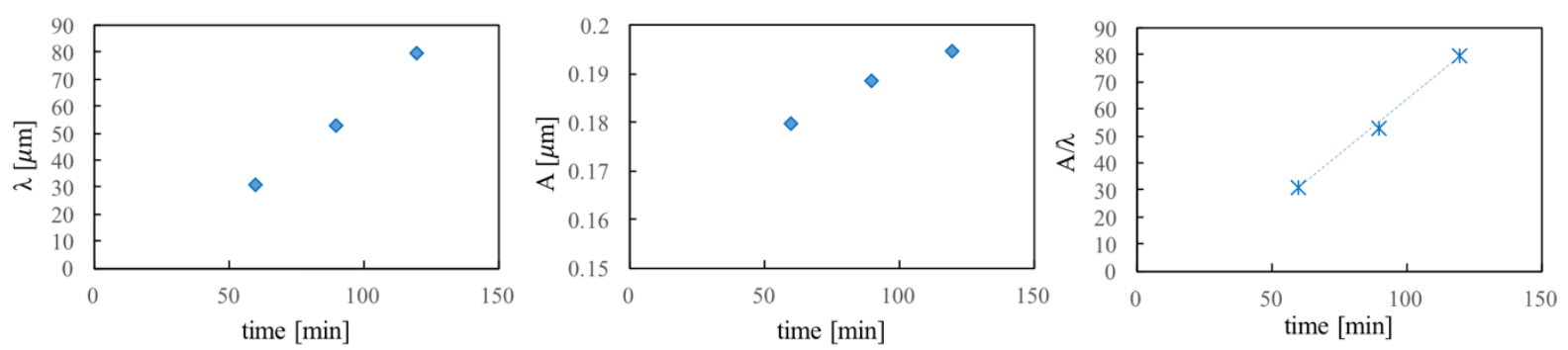

Figure SI10: Wrinkling wavelength, amplitude and aspect ratio (A/ $\lambda$ ), for PDMS oxidised by means of UVO exposure, as a function of time. By contrast with Figure SI8, the aspect ratio does not reach a constant value, indicating that the saturation of the oxide layer is not achieved under these conditions. 


\section{Data interpretation using trilayer model and bilayer models}

We show that, upon increasing PDMS oxidation, a trilayer model should be used to relate wrinkling profile to the film's mechanical properties, since a distinct intermediate oxide layer is formed. For comparison, we also compute the apparent elastic modulus obtained using the bilayer model as a function of substrate oxidation, as shown in Figure SI4. Evidently, the use of the bilayer model when the PDMS oxidised layer is not negligible results in a consistent error in the calculation of the mechanical properties of the film of interest, kept constant in all experiments.

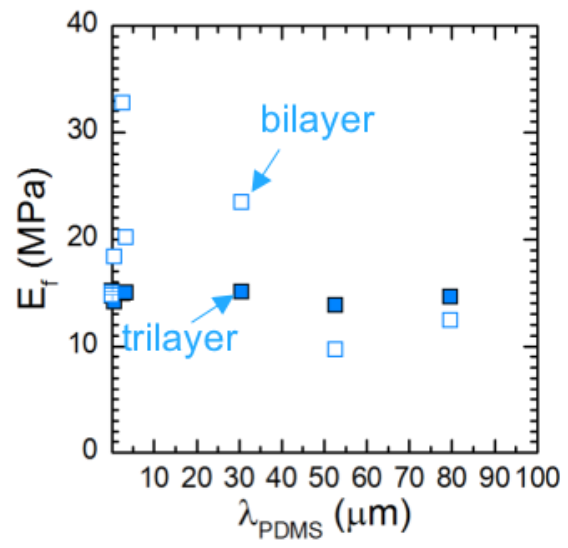

Figure SI11: Comparison of the elastic modulus calculated using the trilayer model (a) and the bilayer model ( $\square$ ) to interpret wrinkling data of the same sample $\left(\mathrm{c}_{0, \mathrm{glycerol}}=2 \mathrm{wt} \%, \mathrm{t}=12 \mathrm{~min}, \mathrm{~T}=25{ }^{\circ} \mathrm{C}\right.$ and $\left.\mathrm{RH}=25 \%\right)$ supported by PDMS substrates oxidised to different extents. The trilayer model provides robust results throughout the whole range while the simpler, bilayer, model yields accurate moduli at the lowest oxidation values of the substrate (when the oxide thickness becomes negligible).

\section{References:}

(1) Rowsell, J.; Nazar, L. F. Speciation and Thermal Transformation in Alumina Sols: Structures of the Polyhydroxyoxoaluminum Cluster $[\mathrm{Al} 30 \mathrm{O} 8(\mathrm{OH}) 56(\mathrm{H} 2 \mathrm{O}) 26] 18+$ and Its $\delta$ Keggin Moieté. Journal of the American Chemical Society 2000, 122 (15), 3777-3778.

(2) Laden, K. Antiperspirants and Deodorants; Second Edition ed.; Marcel Dekker, Inc.: New York. Basel, 1999; Vol. 20. p 404.

(3) Teagarden, D. L.; White, J. L.; Hem, S. L. Aluminum chlorohydrate III: Conversion to aluminum hydroxide. Journal of Pharmaceutical Sciences 1981, 70 (7), 808-810.

(4) Pophristic, V.; Klein, M. L.; Holerca, M. N. Modeling Small Aluminum Chlorohydrate Polymers. The Journal of Physical Chemistry A 2004, 108 (1), 113-120.

(5) Bayley, A.; Liao, J.; Stavrinou, P.; Chiche, A.; Cabral, J. T. Wavefront kinetics of plasma oxidation of polydimethyl siloxane: limits for sub-micron wrinkling. Soft Matter 2014, 10, 1155-1166.

(6) Nania, M.; Matar, O. K.; Cabral, J. T. Frontal vitrification of PDMS using air plasma and consequences for surface wrinkling. Soft Matter 2015,11, 3067-3075. 